\title{
Roles of bimolecular interaction and relative diffusion rate in membrane structure control
}

\author{
Tai-Horng Young ${ }^{\mathrm{a}, *}$ and Leo-Wang Chen ${ }^{\mathrm{b}}$ \\ ${ }^{a}$ Center for Biomedical Engineering, College of Medicine, National Taiwan University, Taipei 10016 (Taiwan) \\ 'Department of Chemical Engineering, National Taiwan University, Taipei 10764 (Taiwan)
}

(Received November 16, 1992; accepted in revised form March 11, 1993)

\begin{abstract}
An attempt was made to elucidate the mechanism of membrane formation and structure control. Ethylene-vinyl alcohol copolymer (EVAL) membranes were prepared by phase inversion from the DMSO solvent into nonsolvent coagulation baths varying in the nature of nonsolvent. The relationship between the bimolecular interaction, the relative diffusion rate and membrane formation is discussed according to two-step mechanism model. It shows that factors affecting membrane properties are the nature of the nonsolvent and the coagulation process. The influence of these factors was investigated and interpreted in terms of the morphological appearance of the membranes as observed by scanning electron microscopy. This analysis provides a better understanding of the membrane formation techniques.
\end{abstract}

Key words: two-step; toplayer; sublayer; interaction; diffusion

\section{Introduction}

Since the development of asymmetric membranes by Loeb and Sourirajan [1], the field of membrane science and technology has experienced an increasing growth. From the technological point of view, the most important properties of membranes are their functional properties such as permeability and permselectivity, which are closely related to their morphological properties [2-6]. For example, much attention has been paid to the design of synthetic polymer membranes made of biomaterials with good compatibility, controlled permeability of water and selectivity. Many techniques have been used to combine mem-

\footnotetext{
*To whom correspondence should be addressed.
}

brane technology with living biological cells in order to treat specific diseases, e.g. the artificial pancreas $[7,8]$. The membrane is permeable to small molecules such as glucose or insulin but totally impermeable to large molecules such as antibodies and lymphocytes. Therefore, it is necessary to prepare membranes with a proper pore size and distribution to meet the requirements for application. Several authors provided models to rationalize the membrane preparation procedure. Strathmann et al. [2] studied the effect of the rate of precipitation on the morphology of symmetric "skin type" membrane. Yao et al. [3] showed that the membrane structure can be predicted by a titration procedure, i.e. a "coagulation value". Uragami et al. [4] showed that there are several solvent groups with different characteris- 
tics, which are classified by their solubility parameters. In our previous studies [9-13], a twostep model [10] was proposed to explain and control the membrane formation mechanism. Following this model, simple and efficient methods used to control membrane morphology are combined and studied in the present paper. Changes in the nonsolvent mixture and the type of coagulation process were found to play a major role under the specified conditions.

\section{Experimental}

\section{Materials}

The ethylene-vinyl alcohol (EVAL) copolymer with a content of $56 \mathrm{~mol} \%$ vinyl alcohol monomer was obtained using previously reported techniques [9]. De-ionized and ultrafiltrated water were used in our experiments. Other reagents were of chemical reagent grade and used without further purification.

\section{Membrane preparation}

The EVAL copolymers were dissolved in dimethyl sulfoxide (DMSO) to form a $25 \%$ polymer solution at $60^{\circ} \mathrm{C}$. The solution was kept at $25^{\circ} \mathrm{C}$ for $24 \mathrm{hr}$ and then cast on glass plates in a uniform thickness of ca. $175 \mu \mathrm{m}$ at $25^{\circ} \mathrm{C}$. The glass plates were immersed immediately (no evaporation step) into coagulation baths with different nonsolvent (NS) compositions at $25^{\circ} \mathrm{C}$ unless stated otherwise. Factors which change the nature of the coagulation bath, including the effects of nonsolvent composition, residence time in the coagulation bath, size of the nonsolvent molecules, and temperature of the coagulation bath, are considered to control the membrane structure. After the polymer had precipitated, the membranes were removed from the glass plate and stored in a pure water bath. The morphology of the membranes was examined with scanning electron microscopy
(SEM). For every SEM picture the composition of the coagulation bath is given in the figure caption.

\section{Theory}

The membranes are produced by the so-called "phase inversion" technique [14]. In this process, a casting solution consisting of a polymer and a solvent is immersed into a nonsolvent coagulation bath. Interchange of solvent and non solvent due to diffusion causes the casting solution to go through a phase transition, by which the membrane is formed. The main route of this process involves two different types of phase transition [10], which can be illustrated in a ternary phase diagram (see Fig. 1). These are: (i) liquid-liquid phase separation, in which the completely miscible solution crosses the binodal boundary to enter the two-phase region (from I to II), and (ii) solidification (from I to II or from II to III); since the viscosity of the polymer solution increases to a certain assumed value, the mobility of the polymer chains will only have limited influence in specifying the morphology.

It has been suggested that the membrane formation process may be described by a two-step mechanism model [10]. The membrane can be

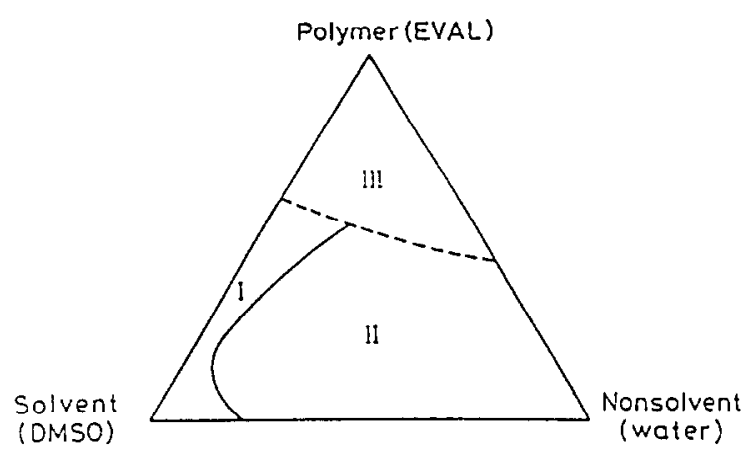

Fig. 1. Ternary phase diagram of EVAL-DMSO-water system. (I) homogeneous solution region, (II) two-phase region, (III) solidification region (data were taken from Ref. [9]). 
divided into two layers: toplayer and sublayer. When the casting solution is immersed into the coagulation bath, the toplayer is formed first at the casting solution-nonsolvent interface. The ratio of nonsolvent inflow to solvent outflow is of utmost importance in the formation of the toplayer structure. If the solvent rapidly diffuses out the casting solution directly enters the solidification region (from I to III) and a dense skin is formed. On the other hand, if the casting solution crosses the binodal to cause liquid-liquid phase separation, the result is a porous toplayer. The structure of the sublayer depends on the local composition at the instant of phase transition. However, it is directly influenced by the degree of aggregation of the polymer molecules at the toplayer. If the toplayer structure is relatively compact, it will increase the barrier for diffusion of solvent from and of nonsolvent into the sublayer. Therefore, the dense toplayer determines the overall diffusion rate through the film and the structure of the sublayer. On the other hand, if the toplayer structure is microporous, the mass transfer rate is only slightly influenced by the toplayer. As a consequence, the sublayer structure is the same as the toplayer structure and the two-step mechanism is reduced to a one-step mechanism.

The local compositional variation of the ternary system is very complex. Young and Chen proposed a simplified diffusion-controlled model for wet-casting membrane formation [11], which allowed the measurement of the compositional variation depending on the flux ratio of solvent to nonsolvent. It was found [12] that the equilibrium amount of nonsolvent $\mathrm{ab}$ sorbed in dense EVAL membranes and the heat of mixing of nonsolvent and DMSO could be used to predict the flux ratio. The equilibrium amount absorbed represents the interaction between EVAL and nonsolvent on one hand, and the tendency of diffusion of nonsolvent into the casting solution on the other. In the same way, the heat of mixing of DMSO and nonsol- vent represents the interaction between solvent and nonsolvent on one hand, and the diffusion tendency of solvent into the coagulation bath on the other.

From the results presented, the different morphological structures of the membranes, either finger-like, dense, or sponge-like, can be obtained. Examples are shown in Table 1. The data in Table 1 has been recently published [12]. The effects of water, acetone and ethanol on EVAL membrane structure are outlined below.

Using water as a coagulation agent gives a dense skin with a finger-like structure, i.e. an asymmetric membrane. Due to the strong interaction between DMSO and water, DMSO in the casting solution desolvates rapidly into the water coagulation bath at the moment the casting solution and water come into contact. However, relatively little water diffuses into the casting solution since it is a medium nonsolvent for the EVAL copolymer. Consequently, EVAL molecules at the interface aggregate rapidly to form a dense skin without pores. As shown in Fig. 1, the casting solution enters the solidification region directly.

The diffusion of DMSO into the water coagulation bath from the sublayer solution is much slower than from the toplayer, because DMSO molecules must diffuse through the dense skin layer. As a result, the composition of the sublayer does not change as rapidly and

\section{TABLE 1}

Properties of representative common nonsolvents for EVAL membrane formation (SEM pictures were presented in Ref. [12], Figs. 2-4.)

\begin{tabular}{llll}
\hline & $\begin{array}{l}\text { Interaction } \\
\text { DMSO/NS }\end{array}$ & $\begin{array}{l}\text { Nonsolvent } \\
\text { for EVAL }\end{array}$ & $\begin{array}{l}\text { Membrane } \\
\text { structure }\end{array}$ \\
\hline $\begin{array}{l}\text { Water } \\
\text { Acetone }\end{array}$ & $\begin{array}{l}\text { Strong } \\
\text { Weak }\end{array}$ & $\begin{array}{l}\text { Middle } \\
\text { Strong }\end{array}$ & $\begin{array}{l}\text { Skin/Finger } \\
\text { Dense }\end{array}$ \\
\hline
\end{tabular}


the solution is within the initial stable homogeneous region.

Water is a medium nonsolvent, so some water molecules diffuse into the interior portion of the fresh solution through the dense skin layer. Since water-DMSO contacts are favored over DMSO-EVAL contacts, finger-like cavities are clearly observed in the membrane where EVAL molecules are clustered together to exclude as much of the DMSO molecules as possible from within their domains.

Because of the weak interaction between DMSO and acetone, DMSO diffuses out slowly with acetone as a nonsolvent. Since acetone is a strong nonsolvent for EVAL, one would not expect a strong nonsolvent to be able to permeate appreciably into the casting solution. Therefore, the process is essentially the same as that using water as the nonsolvent, where the casting solution enters the solidification region directly to form a dense skin at the surface.

Since acetone is a strong nonsolvent almost no acetone molecules can permeate the dense skin to induce liquid-liquid phase separation of the interior casting solution. Thus, the interior casting solution also enters the solidification transition. As a result, a homogeneous dense membrane is formed; the toplayer and the sublayer can be considered as one layer.

The diffusion rate of DMSO into the ethanol coagulation bath is also small owing to the weak interaction between DMSO and ethanol. However, ethanol, a weak nonsolvent, does not act satisfactorily as a strong or medium nonsolvent. Hence the diffusion rate of ethanol into the casting solution is higher than that of water and acetone. The result is that the toplayer of the casting solution maintains a dispersed state and does not precipitate rapidly and compactly. In other words, it forms a porous surface.

The diffusion rate of DMSO from the underlying solution is about the same as that from the surface solution since the porous surface does not greatly influence the diffusion rate.
Ethanol may enter this region by diffusion through the upper porous surface and induce liquid-liquid phase separation. In this way the resulting membrane is porous with the spongelike structure. However, due to the absence of the strong interaction between ethanol and DMSO, when ethanol is used as coagulant, no finger-like cavities will be produced.

\section{Results and discussion}

\section{Effect of nonsolvent mixture: water and acetone}

The strong interaction between solvent and nonsolvent is clearly reduced by adding acetone to the water coagulation bath. Thus the DMSO outflow decreases, phase transition is delayed, and water has more time to diffuse into the surface solution to induce the liquid-liquid phase separation in the toplayer. Figure 2 shows that some pores occur in the toplayer of membrane WA31, but it also has a skin structure. In a manner similar to that observed for the toplayer, DMSO and the nonsolvent mixture do not have an interaction strong enough to expel EVAL molecules; thus the sublayer can not form complete finger-like structures as do membranes coagulated in a water bath.

Basically, using the acetone coagulation bath, the nonsolvent does not permeate into the casting solution, but penetration of nonsolvent into the casting solution can be enhanced by adding water to the coagulation bath. This results in water movement associated with acetone transfer. Therefore, membrane WA13 (Fig. 3) which has a porous toplayer is different from membranes coagulated in only an acetone bath. As mentioned above a porous toplayer has voids through which nonsolvent can pass. So the sublayer goes through the same phase transition and forms a sponge-like structure since the porous surface does not influence diffusion rate of the nonsolvent molecules. Membrane WA22 


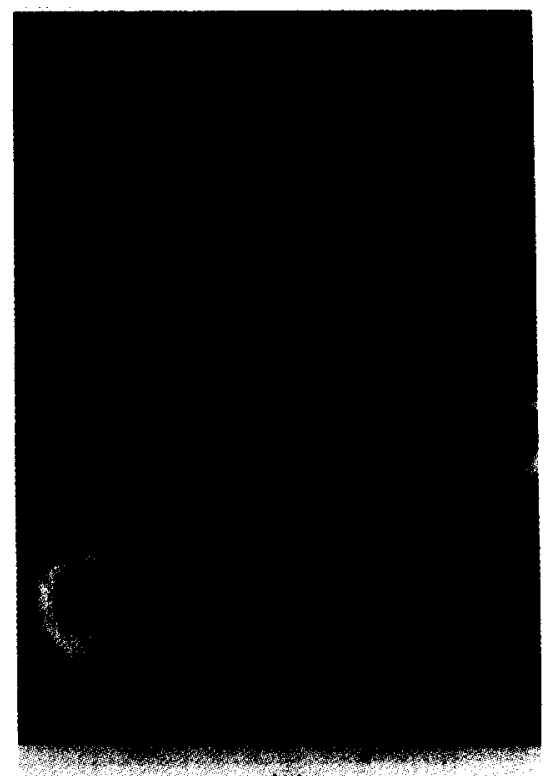

(a)

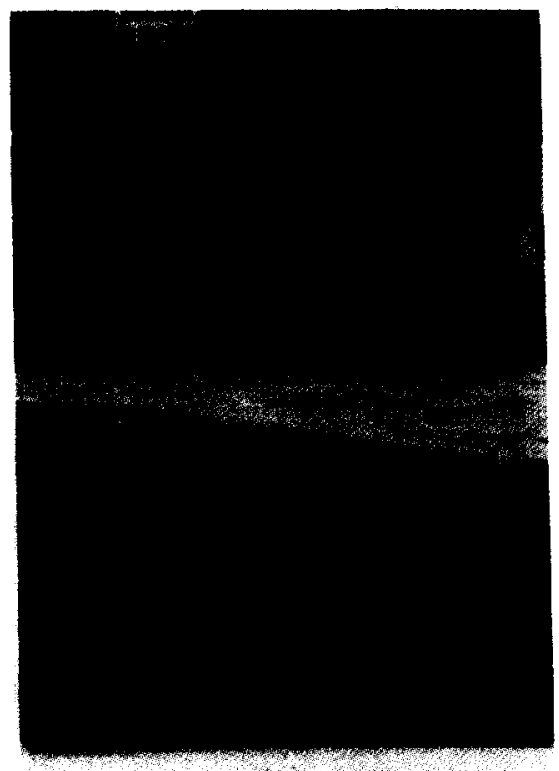

(b)

Fig. 2. SEM pictures of membrane WA31 in a water/acetone bath, containing $75 \%$ water, (a) top and (b) cross section.

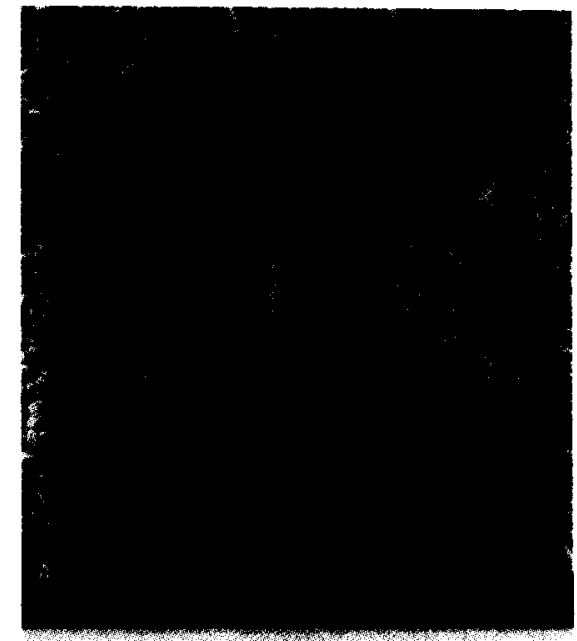

(a)

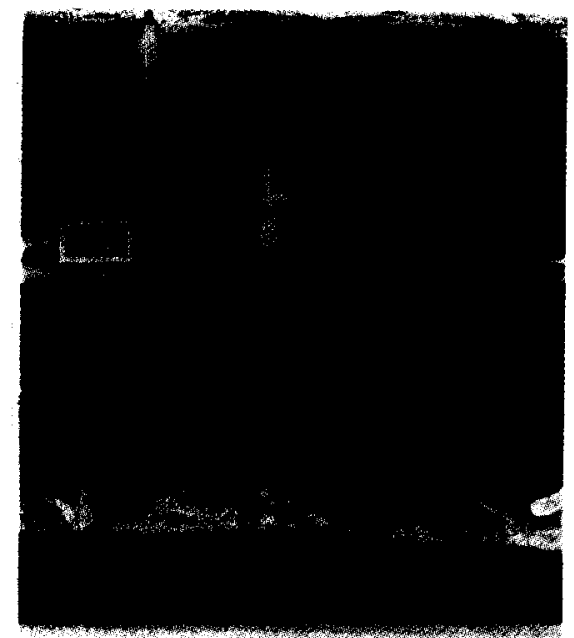

(b)

Fig. 3. SEM pictures of membrane WA13 in a water/acetone bath, containing $25 \%$ water, (a) top and (b) cross section.

(Fig. 4) has a porous surface; the pore distribution is between that of membranes WA13 and WA31. It has a similar spongy sublayer struc- ture as membrane WA13 without any fingerlike structure. This is attributed to the fact that for membranes WA13 and WA22 there is a 


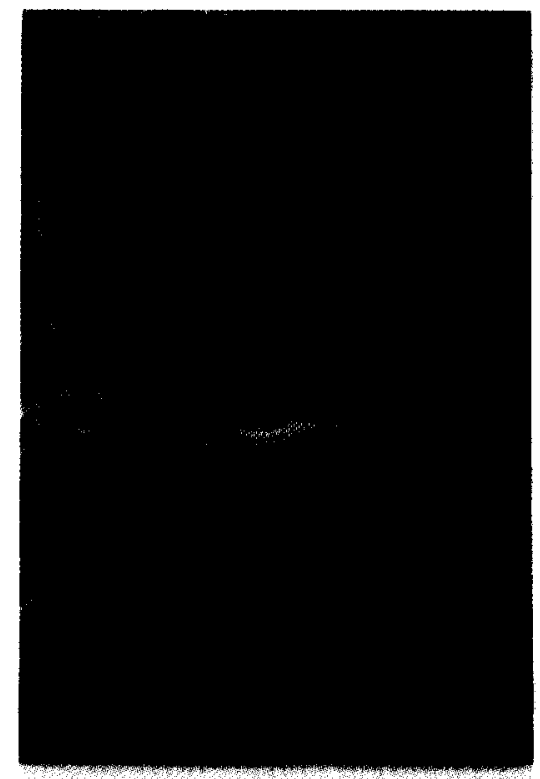

(a)

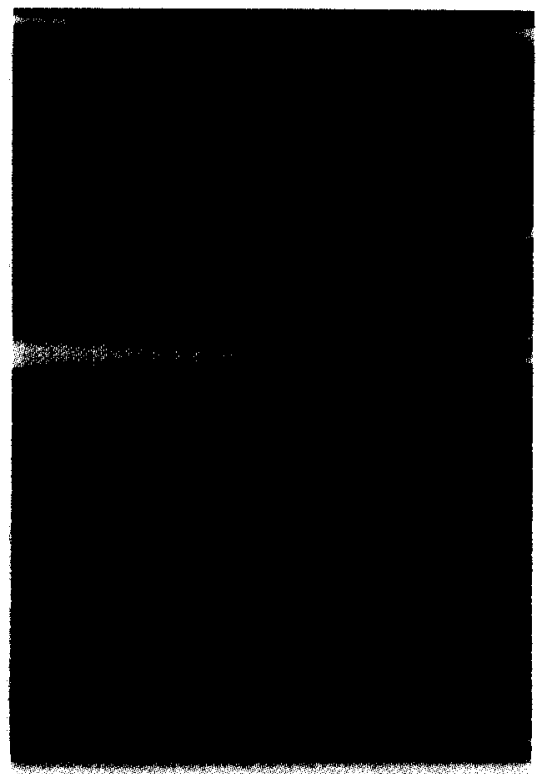

(b)

Fig. 4. SEM pictures of membrane WA22 in a water/acetone bath, containing $50 \%$ water, (a) top and (b) cross section.
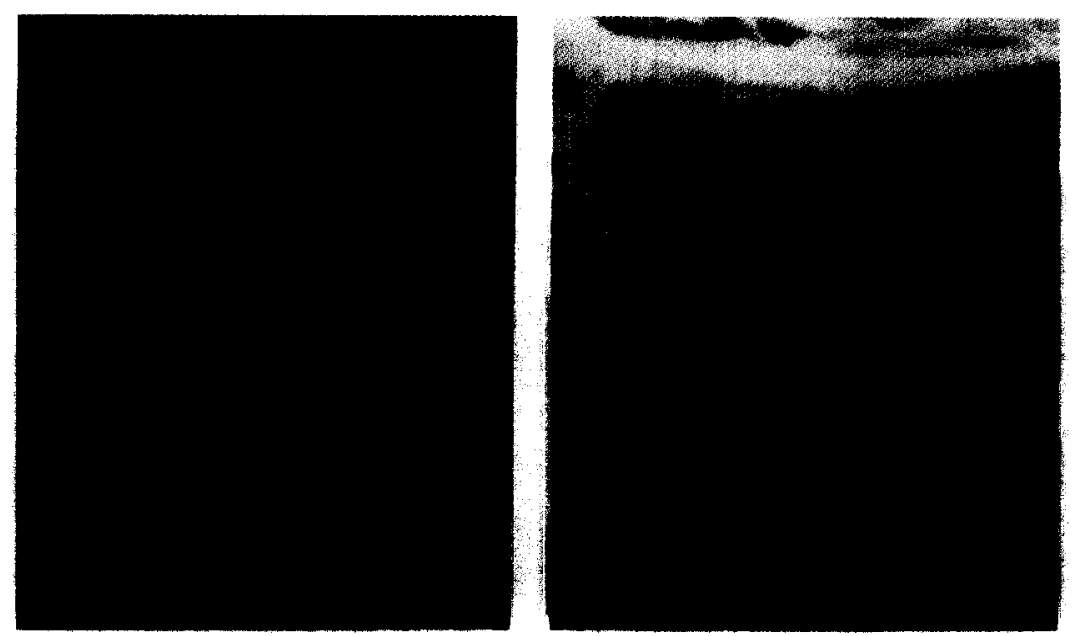

Fig. 5. SEM pictures of membrane WE21 in a water/acetone bath, containing $67 \%$ water, (a) top and (b) cross section.

weaker interaction between solvent and nonsolvent mixture than for membrane WA31. In summary, it is clear that the membrane structure can be modulated by changing the water acetone ratio in the coagulation bath according to the requirements.
Effect of nonsolvent mixture: water and ethanol

When adding ethanol to the water coagulation bath up to a concentration of $33.3 \%$, the overall structure of membrane WE21 is similar 

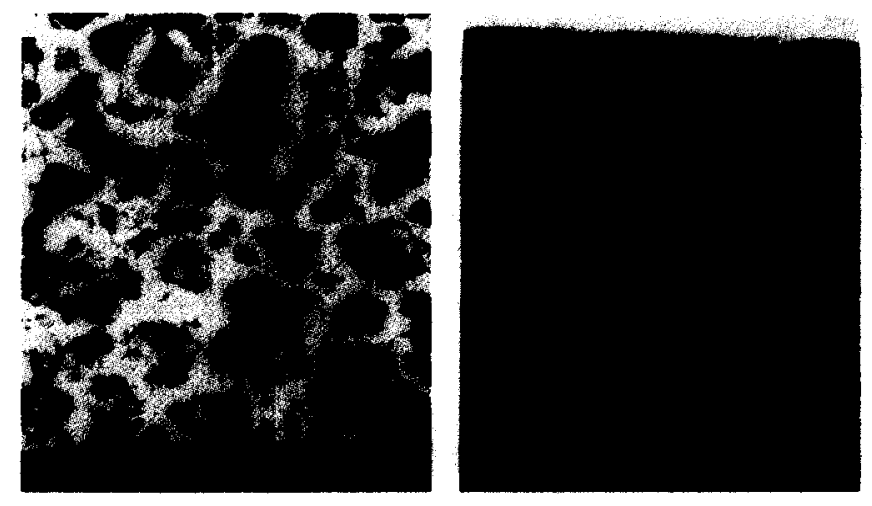

Fig. 6. SEM pictures of membrane WE22 in a water/acetone bath, containing $50 \%$ water, (a) top and (b) cross section.

to that of a membrane coagulated in a pure water bath, but the skin becomes as thin as 0.2 $\mu \mathrm{m}$ (see Fig. 5). When the ethanol content of the coagulation bath is increased to $50 \%$, the dense skin structure disappears and a porous layer occurs in membrane WE22 (Fig. 6). This phenomenon results from the presence of sufficient ethanol, which easier diffuses into the casting solution and thus forms pores in the toplayer. The nonsolvent mixture can now easily diffuse through the porous toplayer into the sublayer. Since the $50 \%$ ethanol content of the coagulation bath reduces the interaction between solvent and nonsolvent mixture, the nonsolvent mixture can not form finger-like structures in the sublayer.

\section{Effect of nonsolvent mixture: acetone and ethanol}

Using acetone as coagulation bath, acetone almost does not diffuse into the casting solution and the resultant membrane has a homogeneous dense structure. Upon adding ethanol to the acetone bath, the outdiffusion rate of DMSO almost does not change, while the indiffusion rate of nonsolvent gradually increases. Therefore, the structure of the resulting membrane becomes less dense. Membrane AE31 has a skin layer with a porous sublayer at an ethanol content of $25 \%$ (Fig. 7). This shows that the diffusion rate of the nonsolvent mixture is slower than that of DMSO so that a dense toplayer is formed. However the diffusion rate of the nonsolvent mixture through the toplayer is faster than that of DMSO and hence a porous sublayer is formed. When the acetone coagulation bath contains $50 \%$ ethanol, the diffusion rate of the nonsolvent mixture into the casting solution increases considerably and a porous toplayer and sublayer are formed. Thus membrane AE22 is a homogeneous membrane (Fig. 8). Membrane AE13 shows the same result and is also a homogeneous membrane (Fig. 9).

\section{Effect of residence time in the coagulation bath}

As another method to control the morphology, coagulation may be carried out in two different baths and regulating the residence time of the casting solution in each bath. In Ref. [11], Fig. 4 shows an example using acetone as nonsolvent in the first coagulation bath followed by immersion in a water bath to form the final membrane. It shows cross-sections of membranes after various residence times in the acetone bath. The thickness of the skin evidently increases with increasing immersion time in the acetone bath. If the immersion time of the casting solution in the first coagulating 


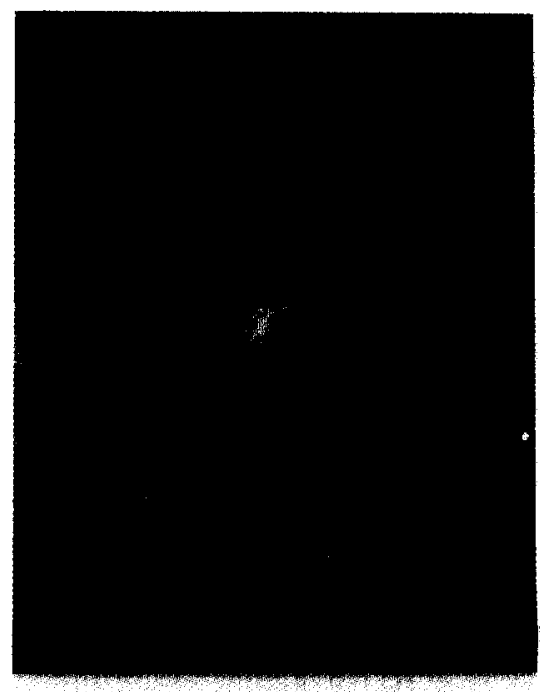

(a)

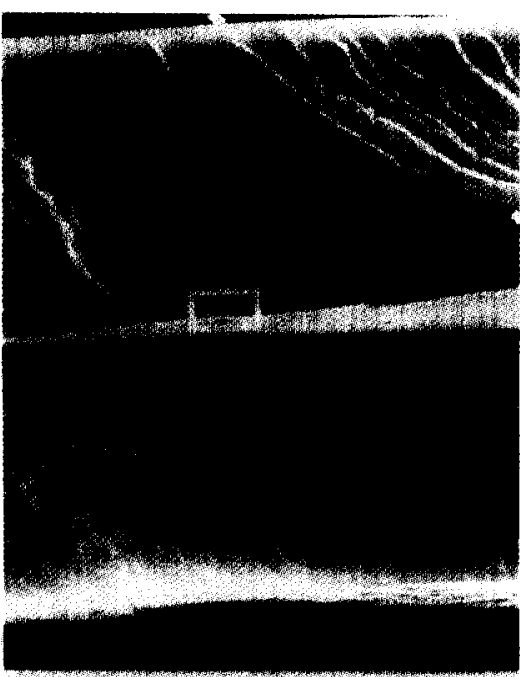

(b)

Fig. 7. SEM pictures of membrane AE31 in a water/acetone bath, containing $75 \%$ acetone, (a) top and (b) cross section.

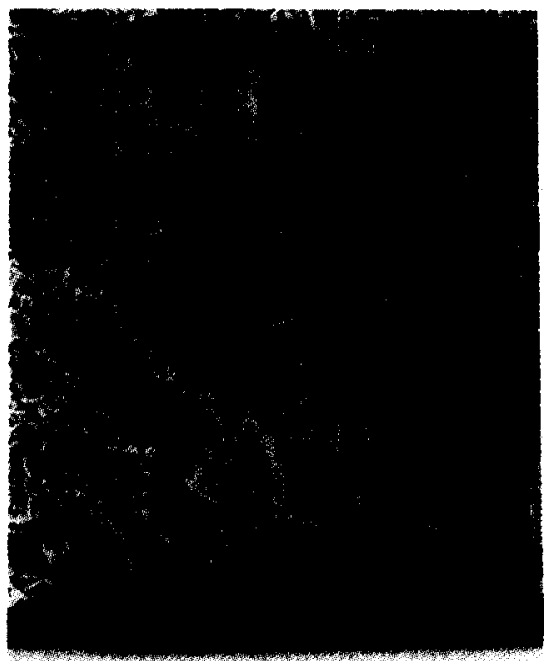

(a)

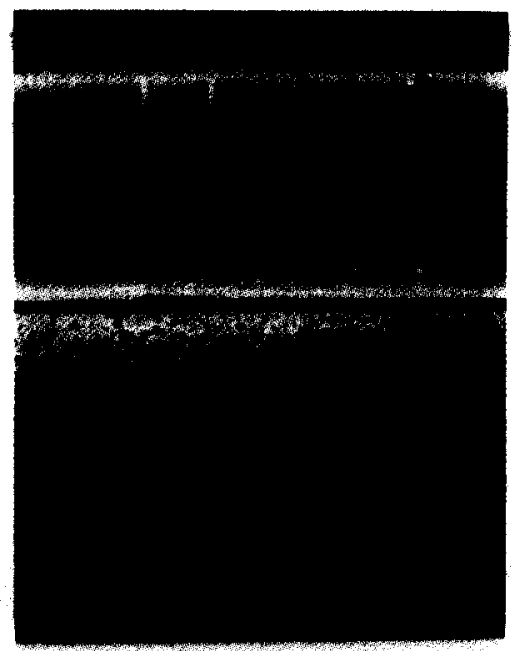

(b)

Fig. 8. SEM pictures of membrane AE22 in a water/acetone bath, containing $50 \%$ acetone, (a) top and (b) cross section.

acetone bath is not long enough to form the overall membrane, the first bath only produces the toplayer. Then the sublayer is formed by using water as the nonsolvent in the second coagulation bath. Since water can diffuse through the upper dense layer, a porous sublayer struc- ture is formed. With increasing the immersion time in the first bath, the sublayer structure changes from finger-type to sponge-type. This results from acetone reducing the interaction between solvent and nonsolvent mixture.

Using ethanol as the nonsolvent in the sec- 

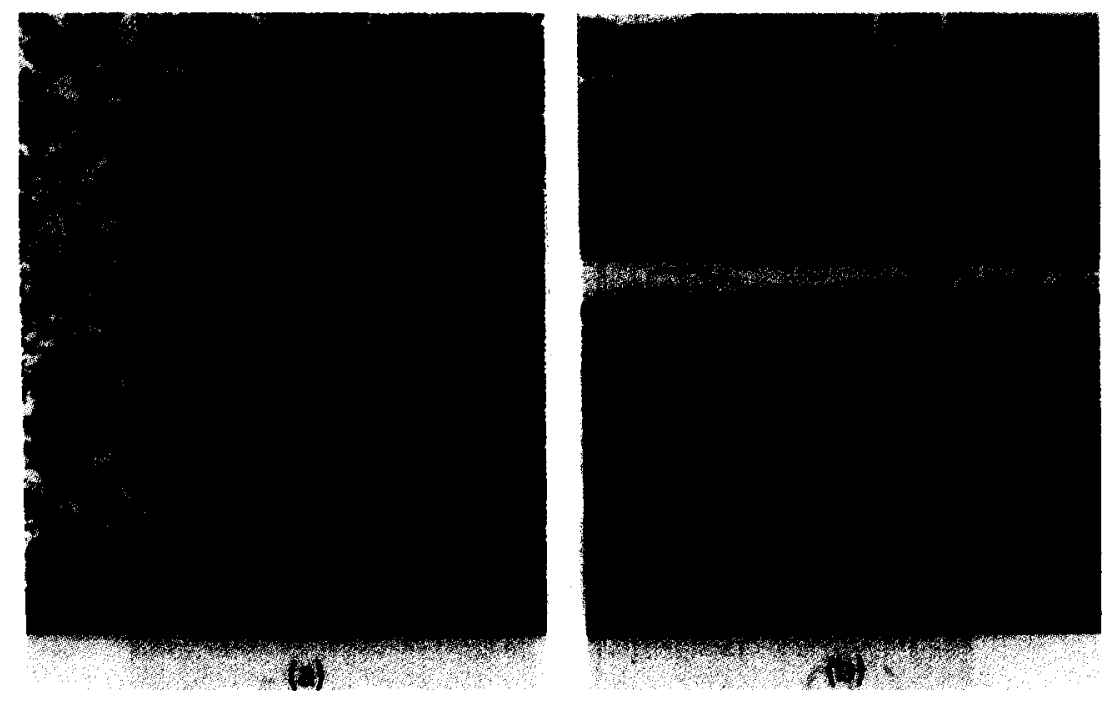

Fig. 9. SEM pictures of membrane AE13 in a water/acetone bath, containing $25 \%$ acetone, (a) top and (b) cross section.

ond coagulation bath, no obvious differences exist between sublayer and toplayer (Fig. 10). It shows that during the overall membrane formation process only DMSO diffuses out. Such a toplayer, i.e., a non-porous skin layer formed in the first acetone bath, has no detectable voids and ethanol passes through such a layer by diffusion as through a solid barrier. Thus the sublayer of the casting solution completely enters the solidification region to form a dense membrane.

Applying the principles described above, the compactness of the dense layer formed in the first acetone bath can regulate the diffusion rate of water and ethanol. The toplayers are almost identical; the main difference is that it is essentially permeable only to water and not to ethanol. Thus, two species with different diffusion rates can be separated by this type of membrane. Such a result can be used to develop membranes for the separation of water/ethanol mixtures by controlling the thickness and compactness of the skin layer.

If the first coagulation bath uses ethanol as nonsolvent, the surface becomes porous due to the nature of ethanol (Fig. 11). So mass transfer can not be controlled by the toplayer. Figures 11(c) and (e) show typical porous toplayers. Figure 11(a) shows a SEM picture of a membrane that has been immersed in the ethanol bath for only one second, so there has not been enough time to form a complete porous structure in the toplayer. However, when coagulated in the ethanol bath first, ethanol has penetrate into the sublayer to reduce the interaction between DMSO and the nonsolvent mixture. So in the second coagulation bath, water that diffuses into the sublayer can not form finger-like structures.

\section{Effect of the size of the nonsolvent molecules}

The factors discussed so far mainly affect the membrane structure, with the toplayer usually forming the main barrier limiting the transmembrane diffusion of molecules. By interposing a toplayer between the nonsolvent and the sublayer, one obviously adds a mass transfer resistance to the system. Whether or not the 

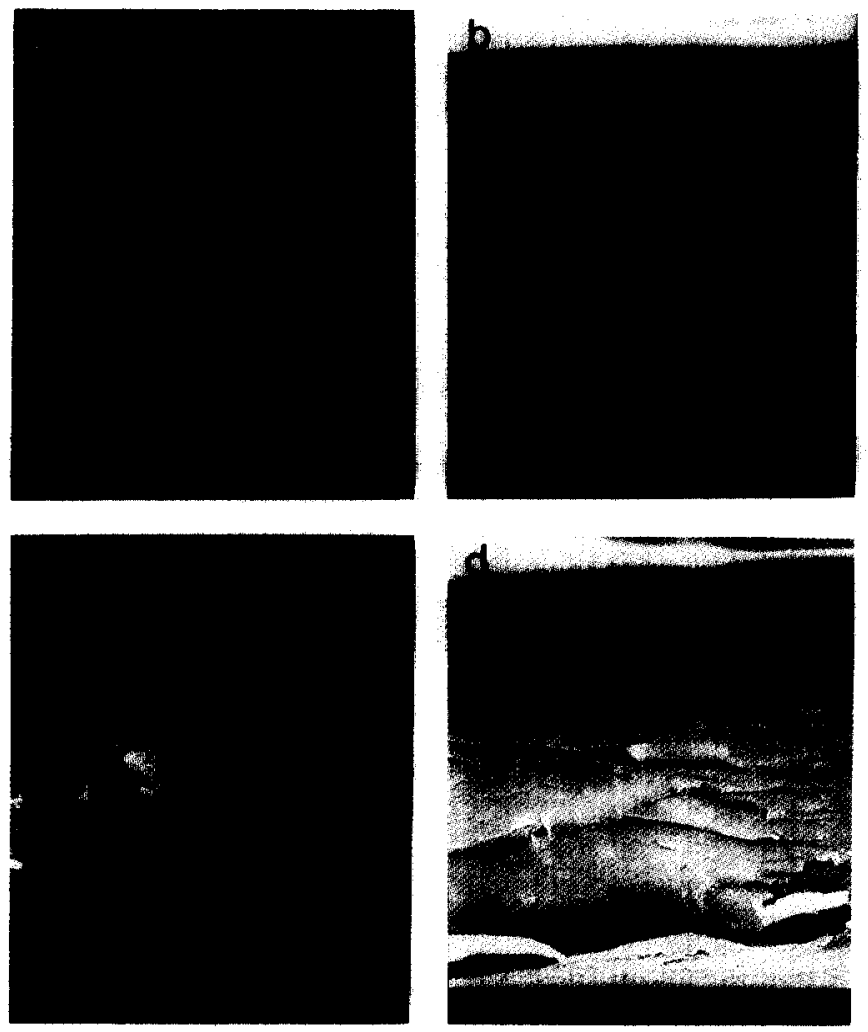

Fig. 10. SEM pictures of EVAL membrane at various time in the acetone bath followed by immersion in the ethanol bath. (a) 5 sec, top section; (b) $5 \mathrm{sec}$, cross section; (c) $10 \mathrm{sec}$, top section; (d) $10 \mathrm{sec}$, cross section.

toplayer seriously impedes solvent and/or nonsolvent transport depends on the nature of the toplayer. Therefore, the toplayer must be designed with mass transport as the key consideration. Another method to control the surface structure is to change the nonsolvent using an alcohol homologous series. A change in stereochemical configuration of the nonsolvent greatly affects its solubility in the polymer. The toplayer structure becomes denser with increasing number of carbon atoms per hydroxyl group (see Fig. 12 and Ref. [12], Fig. 4). This may be attributed to the fact that it is difficult for a nonsolvent with a larger molecular size to permeate into the casting solution, especially into the dense toplayer. So the structure ranges from fairly porous to dense, depending on the molecular size.

\section{Effect of temperature of the coagulation bath}

The structural characteristics of membranes prepared at various temperatures $[9,13]$ of the water coagulation bath are shown below. According to the Le Chatelier-Braun principle, when the mixing of DMSO and water is exoth-

Fig. 11. SEM pictures of EVAL membrane at various time in the ethanol bath followed by immersion in the water bath. (a) $1 \mathrm{sec}$, top section; (b) 1 sec, cross section; (c) 5 sec, top section; (d) 5 sec, cross section; (e) 10 sec, top section; (f) 10 sec, cross section. 

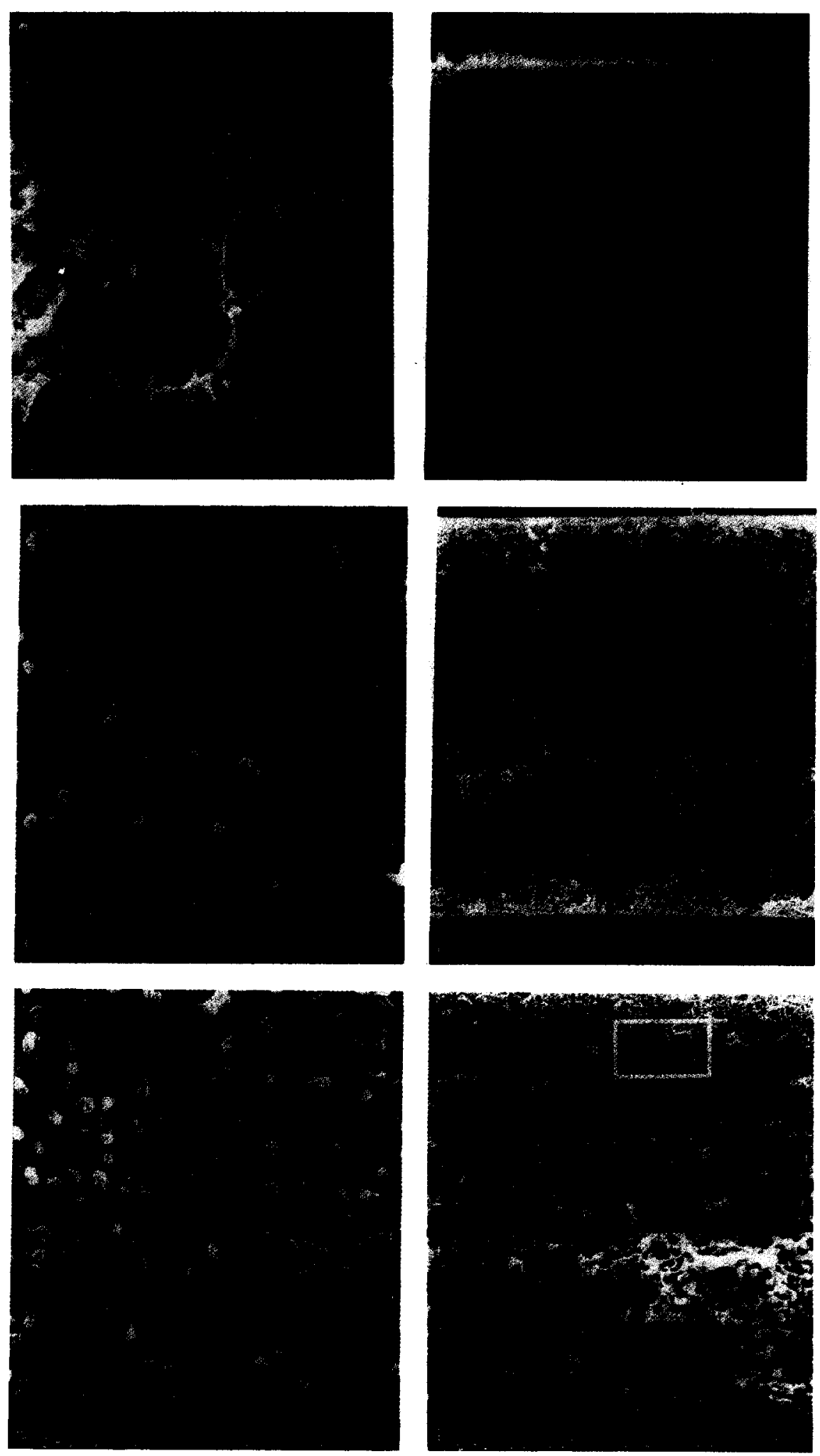

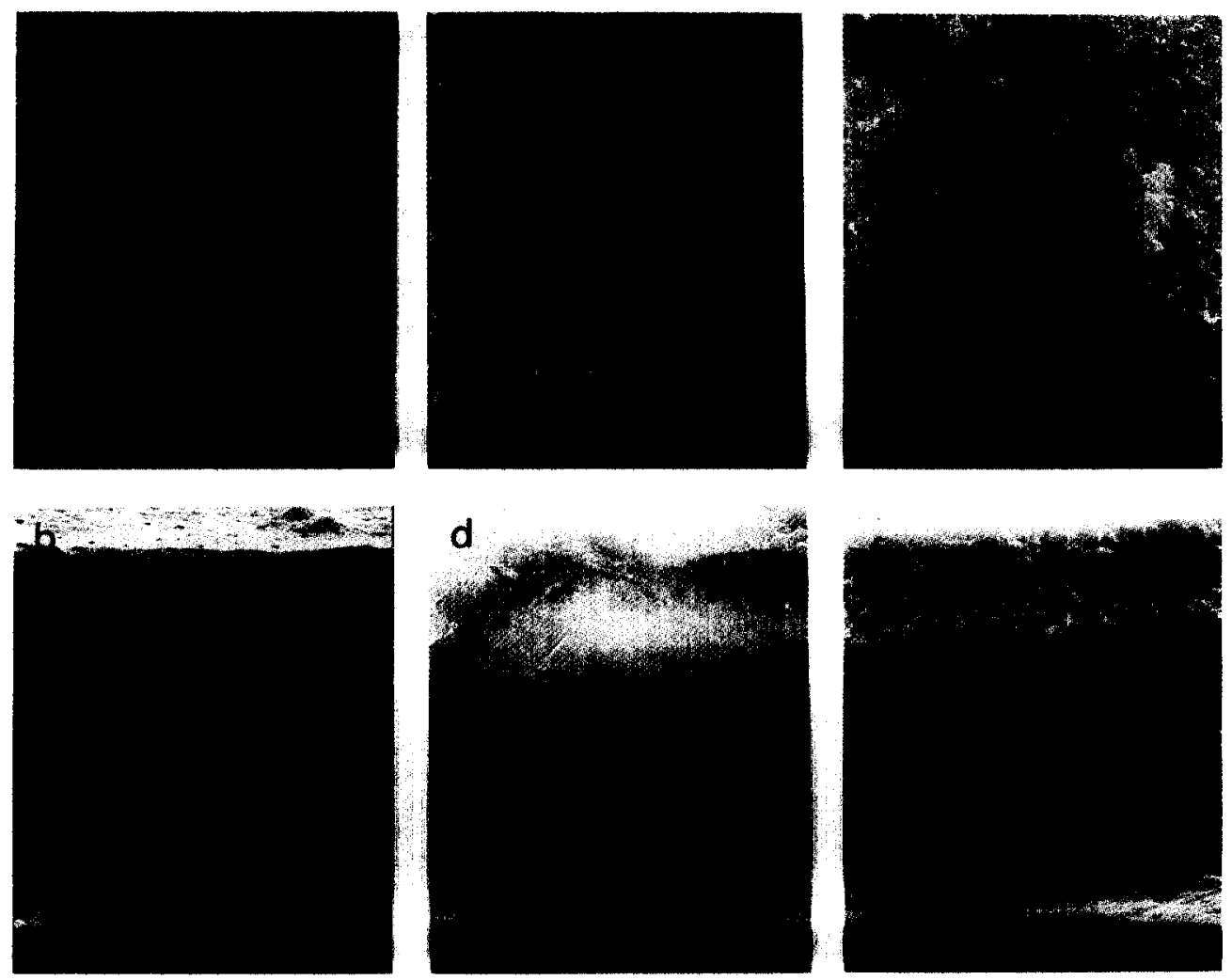

Fig. 12. SEM pictures of EVAL membrane in the different alcohol bath. (a) n-propyl alcohol, top section; (b) n-propyl alcohol, cross section; (c) n-hexyl alcohol, top section; (d) n-hexyl alcohol, cross section; (e) n-octyl alcohol, top section; (f) n-octyl alcohol, cross section.

ermic it may be expected that addition of heat tends to inhibit the mixing reaction, and hence at higher temperatures the outdiffusion rate should decrease. Hence, the viscosity increases less rapidly and it takes more time for the surface solution to undergo phase transition. So more water will diffuse into the surface solution and a porous toplayer will be formed. It can be seen that the pore size and distribution of the membranes increase with increasing temperature of the water bath. For membrane W-60 (Fig. 13), the temperature of the coagulation bath being $60^{\circ} \mathrm{C}$, the mixing tendency is more reduced than for membrane W-45 (Fig. 14), the temperature of the coagulation bath being $45^{\circ} \mathrm{C}$, and so the former only shows the sponge-like structure and does not have enough interaction to form a finger-like structure. The pore size distribution of membrane $W-45$ is also clearly larger than that of a membrane coagulated at $25^{\circ} \mathrm{C}$ (see Ref. [13], Fig. 2).

This result is the same as that obtained with the addition of DMSO solvent into the water coagulation bath [10], which reduces the interaction between the solvent and the nonsolvent mixture. Hence the tendency of DMSO to diffuse into the coagulation bath is reduced and a porous toplayer is formed. Because of the weaker interaction finger-like structures can not be formed in the sublayer. 

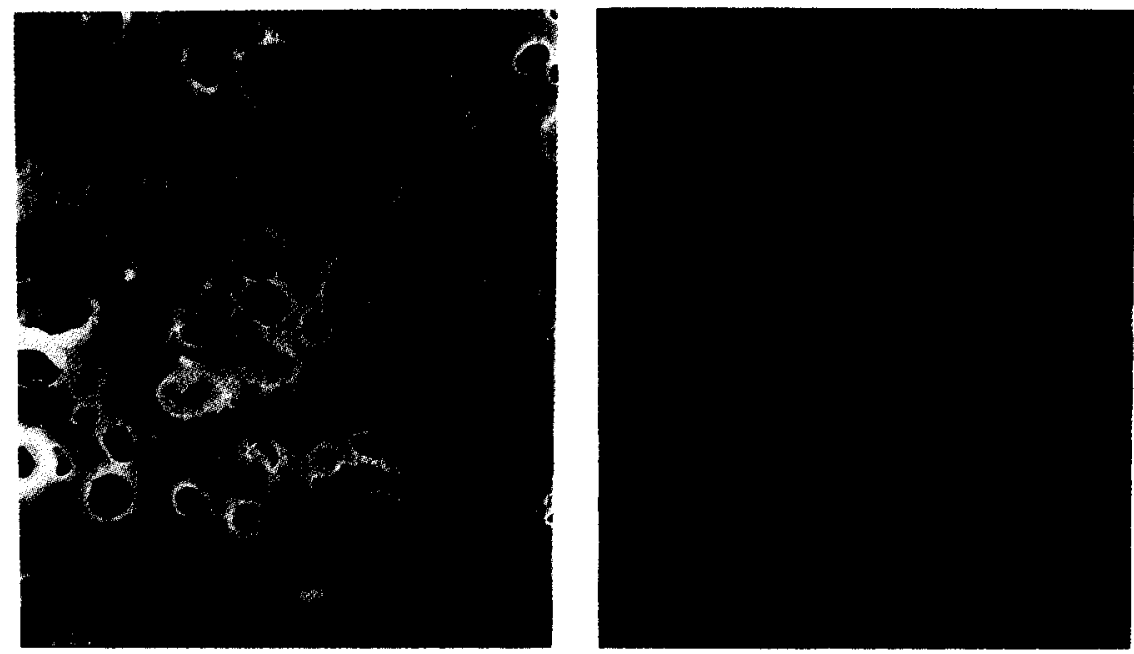

Fig. 13. SEM pictures of membrane $\mathrm{W}-60$ in the water bath at $60^{\circ} \mathrm{C}$, (a) top and (b) cross section.

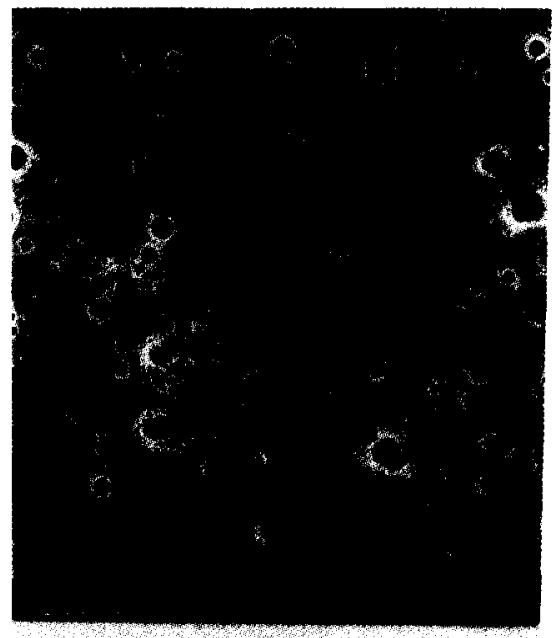

(a)

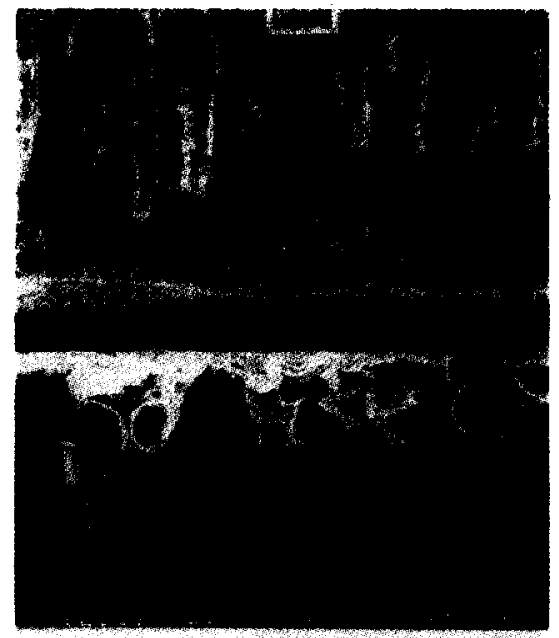

(b)

Fig. 14. SEM pictures of membrane W-45 in the water bath at $45^{\circ} \mathrm{C}$, (a) top and (b) cross section.

\section{Conclusions}

It is clearly shown that using nonsolvent mixtures instead of only one nonsolvent membrane morphology can be more efficiently controlled. This confirms the idea that membrane structure can be successfully explained with bi- molecular interactions and relative diffusion rates. To prevent the formation of a dense structure, the coagulation bath should contain a small amount of a weaker nonsolvent. Nonsolvent mixtures containing a large amount of water will produce finger-like structures. Thus by changing the coagulation conditions, mem- 
branes with different structures, having a wide variety of properties, may be obtained for specific applications.

\section{Acknowledgment}

The authors thank the National Science Council of Taiwan for their financial support of this project.

\section{References}

1 S. Loeb and S. Sourirajan, Sea water demineralization by means of an osmotic membrane, Adv. Chem. Ser., 38 (1963) 117.

2 H. Strathmann, K. Kock and P. Amar, The formation mechanism of asymmetric membrane, Desalination, 16 (1975) 179-203.

3 C.W. Yao, R.P. Burford, A.G. Fane and C.J.D. Fell, Effect of coagulation conditions on structure and properties of membrane from aliphatic polyamides, $\mathrm{J}$. Membrane Sci., 38 (1988) 113-125.

4 T. Uragami, Y. Ohsumi and M. Sugihara, Studies on synthesis and permeabilities of special polymer membranes. 38. Formation mechanism of finger-like cavities in membranes from cellulose nitrate and single solvent, Polymer, 23 (1982) 999-1004.
5 L. Brones, D.M. Koenhen and C.A. Smolders, On the mechanism of formation of asymmetric ultra- and hyper-filtration membranes, Desalination, 22 (1977) 205-219.

6 S Munari, A. Bottino, G.C. Roda and G. Capannelli, Preparation of ultrafiltration membranes. State of the art, Desalination, 77 (1990) 85-100.

7 L.C. Liu, Membrane technology in biomedical engineering, Biomed. Eng. Applications, Basis, Communication, 4 (1992) 365-376.

8 F. Lim and A.M. Sun, Microencapsulated islets as bioartificial endocrine pancreas, Science, 210 (1980) 908-910.

9 L.W. Chen and T.H. Young, EVAL membranes for blood dialysis, Makromol., Chem. Macromol. Symp., 33 (1990) 183-199.

10 T.H. Young and L.W. Chen, A two step mechanism of diffusion controlled ethylene vinyl alcohol membrane formation, J. Membrane Sci., 57 (1991) 69-81.

11 T.H. Young and L.W. Chen, A diffusion-controlled model for wet-casting membrane formation, J. Membrane Sci., 59 (1991) 169-181.

12 L.W. Chen and T.H. Young, Effect of nonsolvent on the mechanism of wet-casting membrane formation from EVAL copolymers, J. Membrane Sci., 59 (1991) 15-26.

13 T.H. Young, Effect of temperature on wet-casting membrane formation from EVAL copolymers, Biomed. Eng.: Applications, Basis, Communication, 4 (1992) 71-78.

14 R.E. Kesting, Synthetic Polymeric Membranes, John Wiley and Sons, New York, NY, 1985. 